\title{
Enzyme-treated Asparagus Extract Down-regulates Heat Shock Protein 27 of Pancreatic Cancer Cells
}

\author{
TAKUYA SHIMADA ${ }^{1}$, YUTA NANIMOTO ${ }^{1}$, BYRON BARON $^{2}$, TAKAO KITAGAWA ${ }^{1}$, \\ KAZUHIRO TOKUDA ${ }^{3}$ and YASUHIRO KURAMITSU ${ }^{1,4}$ \\ Department of ${ }^{1}$ Biochemistry and Functional Proteomics, and \\ ${ }^{3}$ Ophthalmology Yamaguchi University Graduate School of Medicine, Ube, Japan; \\ ${ }^{2}$ Centre for Molecular Medicine and Biobanking, \\ Faculty of Medicine and Surgery, University of Malta, Msida, Malta; \\ ${ }^{4}$ Research Institute of Cancer Prevention, Health Sciences University of Hokkaido, Tobetsu, Japan
}

\begin{abstract}
Background/Aim: From the standpoint of cancer therapy, it is valuable to enhance the anticancer effects of chemotherapy. Our previous reports revealed that upregulation of heat-shock protein 27 (HSP27) has been linked to gemcitabine resistance of pancreatic cancer cells. Enzyme-treated asparagus extract (ETAS) is an extract that is produced from asparagus. The purpose of this study was to investigate the effect of ETAS on the expression of HSP27 and other HSPs in the gemcitabine-resistant pancreatic cancer cell line KLM1-R. Materials and Methods: KLM1-R cells were treated with ETAS, and expression levels of HSPs, including HSP27, were investigated by western blotting. Results: ETAS down-regulated HSP27 and pHSP27 (serine 78) in KLM1-R cells, but, HSP70 and GRP78 levels were not altered. Conclusion: This study suggests the potential therapeutic benefit of ETAS in enhancing anticancer effects by its combination with gemcitabine for patients with pancreatic cancer.
\end{abstract}

Pancreatic cancer is one of the cancers with the poorest prognosis. For patients with advanced pancreatic cancer, gemcitabine (2'-deoxy-2',2'-difluorocytidine monohydrochloride) is the most used chemotherapeutic agent, but intrinsic or acquired resistance disrupts the effects. Our previous study reported that up-regulation of heat-shock protein 27 (HSP27)

This article is freely accessible online.

Correspondence to: Yasuhiro Kuramitsu, MD, Ph.D., Research Institute of Cancer Prevention, Health Sciences University of Hokkaido, Tobetsu-Kanazawa 1757, Hokkaido, Japan. Tel: +81 133231211, Fax: +81 133231669, e-mail: climates@ hoku-iryou.ac.jp

Key Words: HSP27, ETAS, pancreatic cancer, HSP70. was linked to gemcitabine-resistance in pancreatic cancer cells. Conversely, if the expression level of HSP27 can be decreased, the anticancer effect of gemcitabine against gemcitabineresistant pancreatic cancer cells can be re-achieved. In this manuscript the effect of the enzyme treated asparagus extract (ETAS) is reported, which can decrease the expression level of HSP27 in KLM1-R cells in vitro.

ETAS is an extract from asparagus known to induce HSP70 (1). HSP70 is one of the most well-known HSPs. It is a $70-\mathrm{kDa}$ protein expressed in almost all organs (2) such as the gastrointestinal tract (3) and the nervous system (4), and is also known to be involved in tumors $(5,6)$, and neurological diseases (7). From the aspects of these reports, it was obvious that ETAS controls HSP, so this study was carried out to clarify the effect of ETAS on the expression of HSP27 involved in gemcitabine-resistant pancreatic cancer.

\section{Materials and Methods}

Cancer cell line and culture conditions. KLM1-R, a gemcitabineresistant human pancreatic cancer cell line, was kindly provided by the Department of Surgery and Science, Kyushu University Graduate school of Medical Science. KLM1-R was established by exposing gemcitabine to gemcitabine-sensitive KLM1 cells (8). KLM1-R cells were incubated in Roswell Park Memorial Institute (RPMI)-1640 medium with $10 \%$ fetal bovine serum (inactivated at $56^{\circ} \mathrm{C}$ for $30 \mathrm{~min}$ ), and maintained in a humidified $5 \%$ carbon dioxide- $95 \%$ air mixture at $37^{\circ} \mathrm{C}$.

Agents. ETAS and cyclodextrin were kindly provided by the Amino Up Chemical Co., Ltd. (Sapporo, Japan). Cyclodextrin is the excipient for ETAS, and it was used as a control against ETAS. ETAS and cyclodextrin were dissolved in RPMI, diluted, and subjected to filter sterilization treatment.

Sample preparation. ETAS $(0,0.5,1.0,2.0 \mathrm{mg} / \mathrm{ml})$ or cyclodextrin $(2.0 \mathrm{mg} / \mathrm{ml})$ was administered to KLM1-R cells for $120 \mathrm{~h}$. After that, 
cells were collected and homogenized in lysis buffer [50 mM TrisHCL, pH 7.5, $165 \mathrm{mM}$ sodium chloride, $10 \mathrm{mM}$ sodium fluoride, 1 $\mathrm{mM}$ sodium vandate, $1 \mathrm{mM}$ phenylmethylsulfonyl fluoride (PMSF), $10 \mathrm{mM}$ ethylendiaminetetra-acetic acid (EDTA), $10 \mu \mathrm{g} / \mathrm{ml}$ aprotinin, $10 \mu \mathrm{g} / \mathrm{ml}$ leupeptin and $1 \%$ nonylphenoxypolyethoxylethnol-40 (NP40)]. The suspension was further incubated at $4^{\circ} \mathrm{C}$ for $1 \mathrm{~h}$ on a vibrating shaker and centrifuged at $15,000 \times g$ for $30 \mathrm{~min}$ at $4^{\circ} \mathrm{C}$. The supernatants were collected and used for western blotting. Protein concentrations were measured by the Lowry method. Ten samples from KLM1-R were analyzed separately.

Western blot analysis. In order to analyze the expression levels of each protein in KLM1-R cells, $15 \mu \mathrm{g}$ of protein from each sample was analyzed by western blotting. Sodium dodecyl sulfate polyacrylamide gel electrophoresis (SDS-PAGE) was carried out in precast gels (4$20 \%$ gradient acrylamide; Mini-PROTEAN TGX Gels, Bio-Rad. Hercules, CA, USA). After electrophoresis, gels were transferred electrophoretically onto polyvinylidene difluoride membranes (Immobilon-P; Millipore, Bedford, MA, USA) and blocked for $1 \mathrm{~h}$ at room temperature with 5\% skimmed milk dissolved in Tris-buffered saline (TBS). After then, membranes were washed twice with TBS containing $0.05 \%$ Tween-20 and once with TBS.

The primary antibodies used were: mouse monoclonal antibody against HSP27 (dilution 1:200, \#sc-13132 (F-4); Santa Cruz Biotechnology, Santa Cruz, CA, USA), rabbit polyclonal antibody against pHSP27 (serine phosphorylation 78) (dilution 1:200, \#2405; Cell Signaling Technology (CST), Beverly, MA, USA), goat polyclonal antibody against HSP70 (dilution 1:200, \#sc-1060 (K20); Santa Cruz Biotechnology), $78 \mathrm{kDa}$ glucose-regulated protein (GRP78) (dilution 1:200, \#sc-1050 (N-20); Santa Cruz Biotechnology) and actin (dilution 1:200, \#sc-1616 (I-19); Santa Cruz Biotechnology). Membranes were incubated with the primary antibody overnight at $4^{\circ} \mathrm{C}$. They were washed three times with TBS containing $0.05 \%$ Tween- 20 and then incubated with one of the horseradish peroxidase-conjugated secondary antibodies (anti-mouse for HSP27, anti-rabbit for pHSP27 (serine 78), anti-goat for HSP70, GRP78 and actin, dilution 1:10,000; Jackson Immuno Research Laboratories Inc., West Grove, PA, USA) for $1 \mathrm{~h}$ at room temperature. Bands of HSP27, pHSP27 (serine 78), HSP70, GRP78 and actin were visualized by the enhanced chemiluminescence system (ImmunoStar Long Dectection; Wako, Osaka, Japan) and recorded by Image Reader Las-1000 Pro (Fujifilm Corporation, Tokyo, Japan). Expression levels of HSP27, pHSP27 (serine 78), HSP70, GRP78 and actin in the group treated with ETAS $(0.5,1.0$, $2.0 \mathrm{mg} / \mathrm{ml})$ or in the control group $(2.0 \mathrm{mg} / \mathrm{ml}$ of cyclodextrin) in KLM1-R cells and the expression levels of actin in both groups were quantified by analyzing the intensity of each band with Multi Gauge ver 3.0 software (Fujifilm Corporation, Tokyo, Japan).

The statistical significance of differences in HSP27, pHSP27 (serine 78), HSP70, GRP78 and actin expression levels with or without ETAS-treatment in KLM1-R cells were calculated by Student's $t$-test. A $p$-value $<0.05$ was considered to be statistically significant.

\section{Results}

To investigate the effect of ETAS on HSP27, pHSP27 (serine 78), HSP70 and GRP78 expression levels in KLM1-R cells, western blotting with primary antibodies against HSP27, pHSP27 (serine 78), HSP70, GRP78 and actin was performed. The protein expression levels of HSP27 (Figure 1) and pHSP27 (serine 78) (Figure 2) in ETAS-treated KLM $1-\mathrm{R}$ cells were decreased, whereas in the control group, no decrease was observed. However, HSP70 family proteins HSP70 and GRP78 showed the same levels of expression in both groups (Figures 3 and 4).

The ratio of intensities of HSP27/actin and pHSP27/actin in KLM1-R cells were measured. The ratio of the band intensities in the control were considered to be $100 \%$. The ratio of intensities of HSP27/actin, pHSP27 (serine 78)/actin in ETAS-treated or control KLM1-R cells were $55.60 \% \pm 24.30$ (Figure 5) and $75.53 \% \pm 34.89$ (Figure 6), respectively. The ratio of intensity of HSP27/actin, pHSP27 (serine 78)/actin was significantly different $(p<0.05)$ (Figures 5 and 6), but that of HSP70/actin and GRP78/actin did not show any difference (Figures 7 and 8).

These results show that the expression levels of HSP27 and pHSP27 (serine 78) were decreased in KLM1-R cells treated with ETAS.

\section{Discussion}

Heat shock proteins (HSPs), also called stress proteins, are expressed in the cytosol, mitochondria, endoplasmic reticulum, and nucleus and respond to various stresses (physical, chemical, environmental stress) (9). It is known that HSPs function widely in various organisms ranging from humans to bacteria (10). Their main function is to protect the cells and allow cells to survive when exposed to various stresses. This function is an essential function for the survival of living organisms. On the other hand, since they are also expressed in cancer cells, they also bring about resistance to anticancer drugs. Many groups have reported that overexpression of HSP27 in many types of cancer is related to anti-cancer drug resistance and poor prognosis (11-13). In pancreatic cancer cells, our previous study reported that the expression level of HSP27 was elevated in gemcitabine-resistant pancreatic cancer cells compared to gemcitabine-sensitive pancreatic cancer cells $(14,15)$. Therefore, it is thought that HSPs can be targets for anticancer drug treatment (16-19). So far we have reported the trial experiments to down-regulate HSP27 by IFN- $\gamma$, KNK437 or AHCC (20-24). These three materials showed significant synergistic cytotoxic effect on gemcitabine-resistant pancreatic cancer cells by combinatorial treatment with gemcitabine. Since ETAS is an extract from asparagus known to up-regulate HSPs (1), this study was performed to clarify the dynamics of ETAS on HSP27. The results of this study, showed that ETAS suppressed HSP27 expression in vitro. There is a report that ETAS up-regulated HSP 70 (1), and thus the possibility arose that HSP 27 would rise in conjunction with it. However, in this study, the results showed only a decrease 


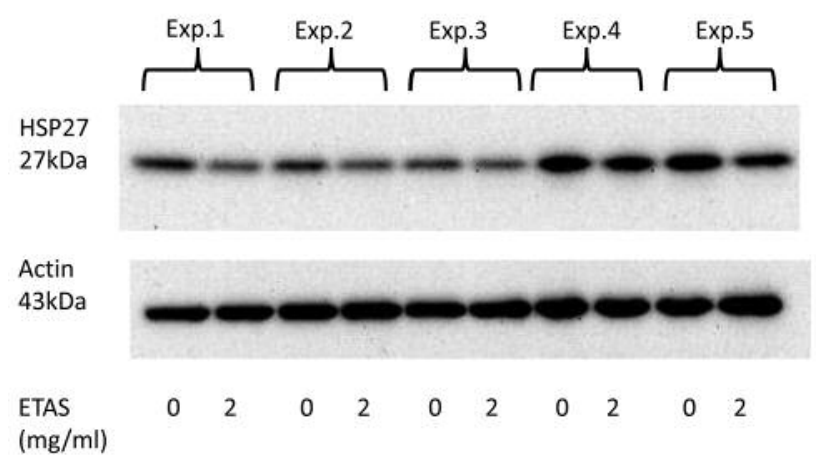

Figure 1. The protein expression level of the heat-shock protein-27 (HSP27) in KLM1-R cells treated with enzyme-treated asparagus extract (ETAS) or cyclodextrin (control). Western blot analysis of HSP27 protein and actin in KLM1-R cells treated with ETAS $(0,2 \mathrm{mg} / \mathrm{ml})$. The protein expression levels of HSP27 (bands of $27 \mathrm{kDa}$ ) of ETAS-treated KLM1-R cells were decreased, whereas in the control group, no decrease was observed. However, actin (bands of $43 \mathrm{kDa}$ ) showed the same levels of expression in both groups.

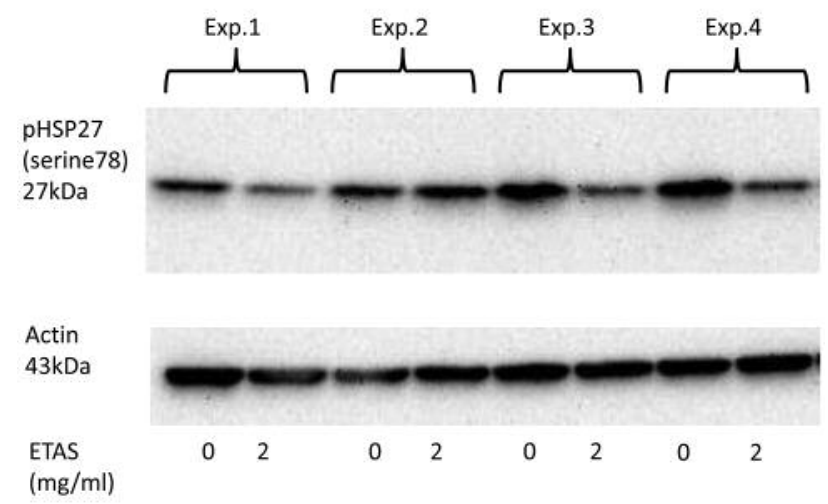

Figure 2. The protein expression level of the heat-shock protein-27 (pHSP27 (serine 78)) in KLM1-R cells treated with enzyme treated asparagus extract (ETAS) or cyclodextrin (control). Western blot analysis of pHSP27 (pHSP27 (serine 78)) protein and actin in KLM1$R$ cells treated with ETAS $(0,2 \mathrm{mg} / \mathrm{ml})$. The protein expression levels of pHSP27 (serine 78) (bands of $27 \mathrm{kDa}$ ) of ETAS-treated KLM1-R cells were decreased, whereas in the control group, no decrease was observed. However, actin (bands of $43 \mathrm{kDa}$ ) showed the same levels of expression in both groups.

in HSP 27 levels, and from the viewpoint of HSP 27, there is a possibility for ETAS to be used in cancer treatment as an auxiliary substance. It may be used especially in the case of pancreatic cancer, where the 5-year survival rate is extremely low as $6.9 \%$, and is one of the cancers (25) with poor prognosis, which usually presents at anwith advanced stage at the time of diagnosis.

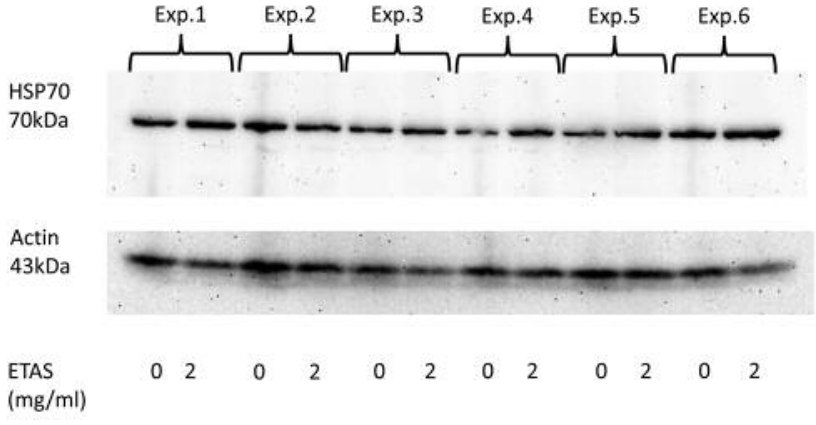

Figure 3. The protein expression level of the heat-shock protein-70 (HSP70) in KLM1-R cells treated with enzyme treated asparagus extract (ETAS) or cyclodextrin (control). Western blot analysis of HSP70 protein and actin in KLM1-R cells treated ETAS $(0,2 \mathrm{mg} / \mathrm{ml})$. The protein expression levels of HSP70 (bands of $70 \mathrm{kDa}$ ) in both ETAS-treated and control group of KLM1-R cells were not changed. Also, actin (bands of $43 \mathrm{kDa}$ ) showed the same levels of expression in both groups.

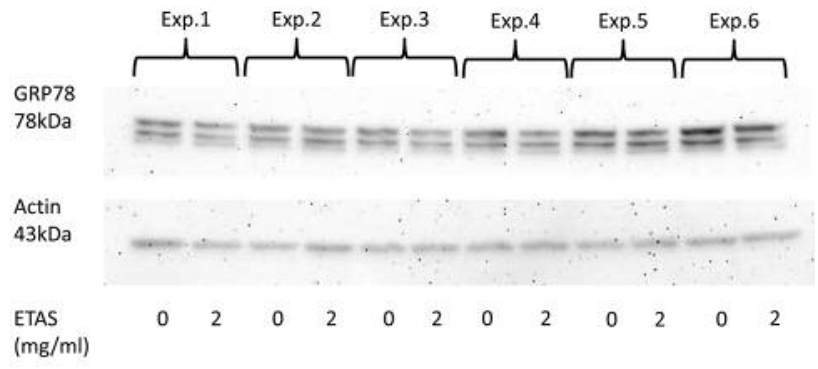

Figure 4. The protein expression level of the glucose regulated protein78 (GRP78) in KLM1-R cells treated with enzyme treated asparagus extract (ETAS) or cyclodextrin (control). Western blot analysis of GRP78 protein and actin in KLM1-R cells treated ETAS $(0,2 \mathrm{mg} / \mathrm{ml})$. The protein expression levels of GRP78 (bands of $78 \mathrm{kDa}$ ) in both ETAS-treated and control group of KLM1-R cells were not changed. Also, actin (bands of $43 \mathrm{kDa}$ ) showed the same levels of expression in both groups.

Many pancreatic cancer patients treated with chemotherapeutic agents show resistance to gemcitabine, the first-choice chemotherapy drug, and they are, thus, difficult to treat. Response efficiency with gemcitabine alone was $13.3 \%$ (26), response rate with gemcitabine and S-1 concomitant administration was $29.3 \%$ (26), and response rate with gemcitabine and nab-paclitaxel concomitant administration was $58.8 \%$ which is not a good result at all (27).

In the future, it is necessary to compare the antitumor effect of the gemcitabine-alone group and ETAS/gemcitabine- 


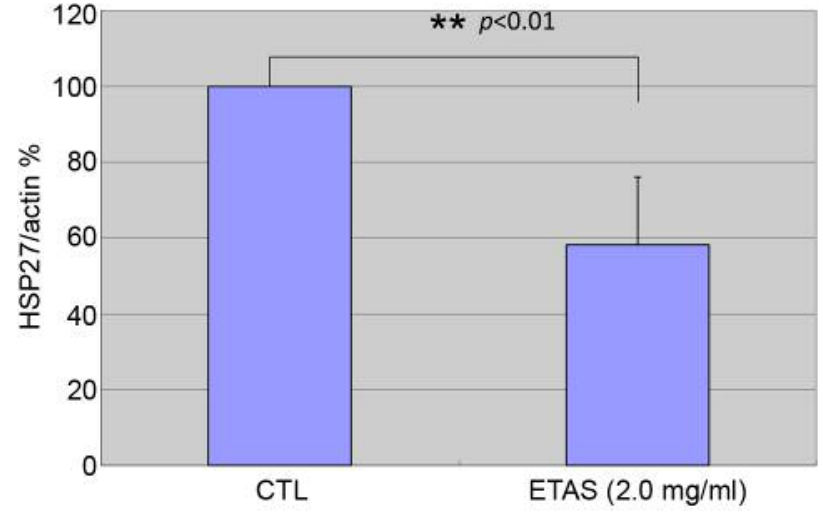

Figure 5. The intensity of the HSP27/actin bands in KLM1-R cells. This graph shows that the ratio of the intensity of HSP27/actin in KLM1-R cells was significantly reduced by ETAS treatment in vitro $(p<0.01$ by the Student's $t$-test $)(n=5)$.

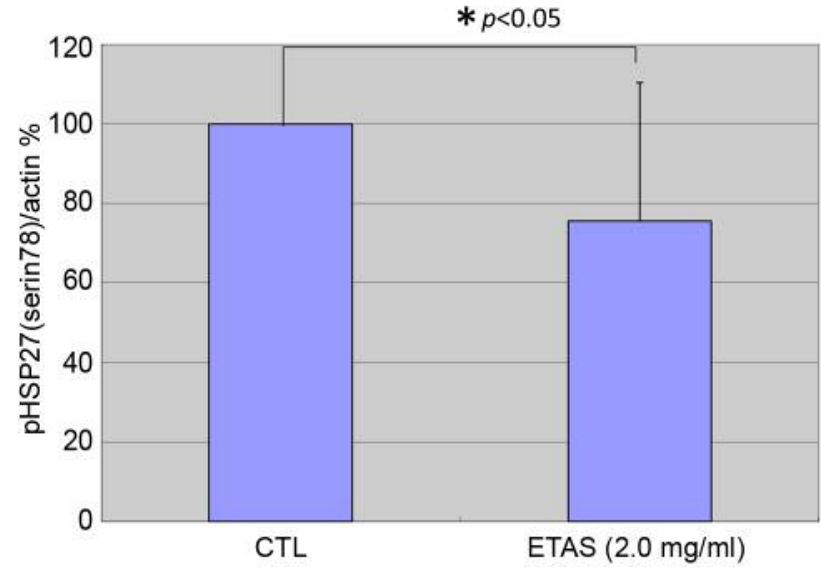

Figure 6. The intensity of the pHSP27 (serine 78)/actin bands in KLM1$R$ cells. This graph shows that the ratio of the intensity of pHSP27 (serine 78)/actin in KLM1-R cells was significantly reduced by ETAS treatment in vitro $(p<0.05$ by Student's $t$-test $)(n=4)$.

combination group against KLM1-R in vitro and in vivo. This study suggested that a synergistic action of ETAS combined with gemcitabine would be expected.

\section{Acknowledgements}

The Authors thank Dr. Shin-ichiro Maehara and Prof. Yoshihiro Maehara at Kyushu University for providing KLM1-R cells. Immunoblot detection by LAS-1000 was conducted at Yamaguchi University Gene Research Center. Regarding this article, there are no conflicts of interest to be disclosed.

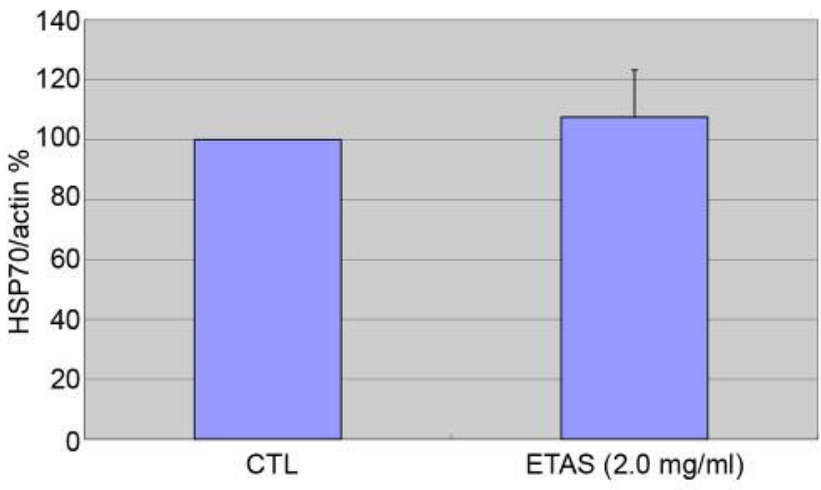

Figure 7. The intensity of the HSP70/actin bands in KLM1-R cells. This graph shows that the ratio of the intensity of HSP70/actin in KLM1-R cells was not significantly reduced by ETAS treatment in vitro $(n=6)$.

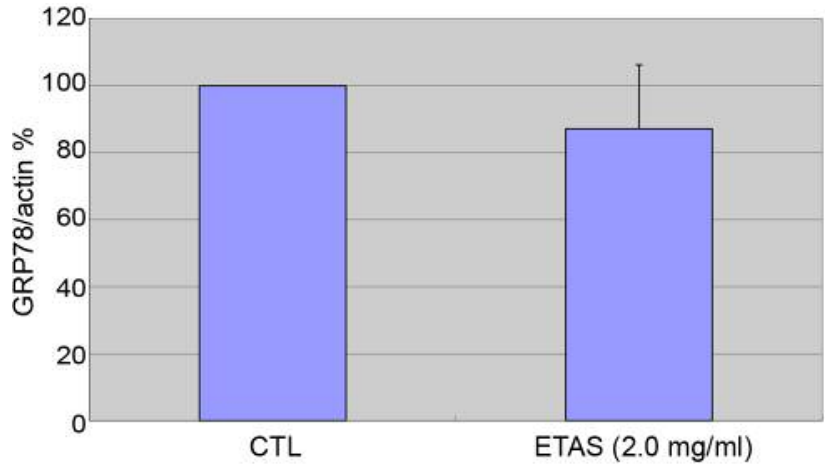

Figure 8. The intensity of the GRP78/actin bands in KLM1-R cells. This graph shows that the ratio of the intensity of GRP78/actin in KLM1-R cells was not significantly reduced by ETAS treatment in vitro $(n=6)$.

\section{References}

1 Ito T, Goto K, Takanari J, Miura T, Wakame K, Nishioka H, Tanaka A and Nishihira J: Effects of enzyme-treated asparagus extract on heat shock protein 70, stress indices, and sleep in healthy adult men. J Nutr Sci Vitaminol (Tokyo) 60: 283-290, 2014.

2 Daugaard M, Rohde $M$ and Jäättelä $M$ : The heat shock protein 70 family: Highly homologous proteins with overlapping and distinct functions. FEBS Lett 581: 3702-3710, 2007.

3 Hirata I, Naito Y, Handa O, Hayashi N, Mizushima K, Adachi S, Omatsu T, Okayama T, Kishimoto E, Ichikawa H, Takagi T, 
Kokura S, Otaka M and Yoshikawa T: Heat-shock protein 70overexpressing gastric epithelial cells are resistant to indomethacin-induced apoptosis. Digestion 79: 243-250, 2009.

4 Sabirzhanov B, Stoica BA, Hanscom M, Piao CS and Faden AI: Over-expression of HSP70 attenuates caspase-dependent and caspase-independent pathways and inhibits neuronal apoptosis J Neurochem 123: 542-554, 2012.

5 Gabai VL, Yaglom JA, Wang Y, Meng L, Shao H, Kim G, Colvin T, Gestwicki J and Sherman MY: Anticancer Effects of targeting Hsp70 in tumor stromal cells. Cancer Res 15: 59265932, 2016.

6 Jagadish N, Parashar D, Gupta N, Agarwal S, Suri V, Kumar R, Suri V, Sadasukhi TC, Gupta A, Ansari AS, Lohiya NK and Suri A: Heat shock protein 70-2 (HSP70-2) is a novel therapeutic target for colorectal cancer and is associated with tumor growth. BMC Cancer 29: 561, 2016.

7 Soo ET, Ng YK, Bay BH and Yip GW: Heat shock proteins and neurodegenerative disorders. Scientific World Journal 8: 270274, 2008.

8 Maehara S, Tanaka S, Shimada M, Shirabe K, Saito Y, Takahashi $\mathrm{K}$ and Maehara Y: Selenoprotein P, as a predictor for evaluating gemcitabine resistance in human pancreatic cancer cells. Int J Cancer 112: 184-189, 2004.

9 Kregel KC: Heat shock proteins: modifying factors in physiological stress responses and acquired thermotolerance. J Appl Physiol 92: 2177-2186, 2002.

10 Schlesinger MJ: Heat shock proteins. J Biol Chem 265: 1211112114, 1990.

11 Kang SH, Kang KW, Kim KH, Kwon B, Kim SK, Lee HY, Kong SY, Lee ES, Jang SG and Yoo BC: Up-regulated HSP27 in human breast cancer cells reduces Herceptin susceptibility by increasing HER2 protein stability. BMC Cancer 8: 286, 2008.

12 Hsu HS, Lin JH, Huang WC, Hsu TW, Su K, Chiou SH, Tsai YT and Hung SC: Chemoresistance of lung cancer stem-like cells depends on activation of Hsp27. Cancer 117: 1516-1528, 2011.

13 Liu CL, Chen SF, Wu MZ, Jao SW, Lin YS, Yang CY, Lee TY, Wen LW, Lan GL and Nieh S: The molecular and clinical verification of therapeutic resistance via the p38 MAPK-Hsp27 axis in lung cancer. Oncotarget 22: 14279-14290, 2016.

14 Mori-Iwamoto S, Kuramitsu Y, Ryozawa S, Mikuriya K, Fujimoto M, Maehara S, Maehara Y, Okita K, Nakamura K and Sakaida I: Proteomics finding heat-shock protein 27 as a biomarker for resistance of pancreatic cancer cells to gemcitabine. Int J Oncol 31: 1345-1350, 2007.

15 Mori-Iwamoto S, Kuramitsu Y, Ryozawa S, Taba K, Fujimoto M, Okita K, Nakamura K and Sakaida I: A proteomic profiling of gemcitabine resistance in pancreatic cancer cell lines. Mol Med Rep 1: 429-434, 2008.

16 Ciocca DR and Calderwood SK: Heat shock proteins in cancer: diagnostic, prognostic, predictive, and treatment implications. Cell Stress Chaperones 10: 86-103, 2005.

17 Garrido C, Brunet M, Didelot C, Zermati Y, Schmitt E and Kroemer G: Heat shock proteins 27 and 70: Anti-apoptotic proteins with tumorigenic properties. Cell Cycle 5: 2592-2601, 2006.

18 Chen L, Li J, Farah E, Sarkar S, Ahmad N, Gupta S, Larner J and Liu X: Cotargeting HSP90 and its client proteins for treatment of prostate cancer. Mol Cancer Ther 15: 2107-2118, 2016.
19 Fernandes J and Alves P: Recent patents on Heat shock proteins targeting antibodies. Recent Pat Anticancer Drug Discov 12: 48$54,2017$.

20 Mori-Iwamoto S, Taba K, Kuramitsu Y, Ryozawa S, Tanaka T, Maehara S, Maehara Y, Okita K, Nakamura K and Sakaida I: Interferon- $\gamma$ down-regulates HSP27 of pancreatic cancer cells, and helps the cytotoxic effect of gemcitabine. Pancreas 38: 224226, 2009.

21 Taba K, Kuramitsu Y, Ryozawa S, Yoshida K, Tanaka T, MoriIwamoto S, Maehara S, Maehara Y, Sakaida I and Nakamura K: KNK437 downregulates HSP27 of pancreatic cancer cells, and helps the cytotoxic effect of gemcitabine. Chemother 57: 12-16, 2011.

22 Kuramitsu Y, Wang Y, Taba K, Suenaga S, Ryozawa S, Kaino S, Sakaida I and Nakamura K: Heat-shock protein 27 plays the key role in gemcitabine-resistance of pancreatic cancer cells. Anticancer Res 32: 2295-2299, 2012.

23 Suenaga S, Kuramitsu Y, Kaino S, Maehara S, Maehara Y, Sakaida I and Nakamura K: Active hexose-correlated compound down-regulates HSP27 of pancreatic cancer cells, and helps the cytotoxic effect of gemcitabine. Anticancer Res 34: 141-146, 2014.

24 Tokunaga M, Baron B, Kitagawa T, Tokuda K and Kuramitsu Y: Active hexose-correlated compound down-regulates heat shock factor 1, a transcription factor for HSP27, in gemcitabineresistant human pancreatic cancer cells. Anticancer Res 35: 6063-6067, 2015.

25 Kuroda T, Kumagi T, Yokota T, Seike H, Nishiyama M, Imai Y, Inada N, Shibata N, Imamine S, Okada S, Koizumi M, Yamanishi H, Azemoto N, Miyaike J, Tanaka Y, Tatsukawa H, Utsunomiya H, Ohno Y, Miyake T, Hirooka M, Furukawa S, Abe M, Ikeda Y, Matsuura B, Hiasa Y and Onji M: Improvement of long-term outcomes in pancreatic cancer and its associated factors within the gemcitabine era: a collaborative retrospective multicenter clinical review of 1,082 patients. BMC Gastroenterol 13: 134, 2013.

26 Ueno H, Ioka T, Ikeda M, Ohkawa S, Yanagimoto H, Boku N, Fukutomi A, Sugimori K, Baba H, Yamao K, Shimamura T, Sho M, Kitano M, Cheng AL, Mizumoto K, Chen JS, Furuse J, Funakoshi A, Hatori T, Yamaguchi T, Egawa S, Sato A, Ohashi Y, Okusaka T and Tanaka M: Randomized phase III study of gemcitabine plus S-1, S-1 alone, or gemcitabine alone in patients with locally advanced and metastatic pancreatic cancer in Japan and Taiwan: GEST Study. J Clin Oncol 31: 1640-1648, 2013.

27 Ueno H, Ikeda M, Ueno M, Mizuno N, Ioka T, Omuro Y, Nakajima TE and Furuse J: Phase I/II study of nab-paclitaxel plus gemcitabine for chemotherapy-naive Japanese patients with metastatic pancreatic cancer. Cancer Chemother Pharmacol 77: 595-603, 2016.
Received April 3, 2018

Revised April 23, 2018

Accepted April 24, 2018 